\title{
User-centered development and testing of a monitoring system that provides feedback regarding physical functioning to elderly people
}

This article was published in the following Dove Press journal:

Patient Preference and Adherence

26 August 2013

Number of times this article has been viewed

\author{
Joan Vermeulen' \\ Jacques CL Neyens' \\ Marieke D Spreeuwenberg' \\ Erik van Rossum ${ }^{1,2}$ \\ Walther Sipers ${ }^{3}$ \\ Herbert Habets ${ }^{3}$ \\ David J Hewson ${ }^{4}$ \\ Luc P de Witte ${ }^{1,2}$
}

'School for Public Health and Primary Care (CAPHRI),

Maastricht University, Maastricht,

The Netherlands; ${ }^{2}$ Research Center

for Technology in Care, Zuyd

University of Applied Sciences,

Heerlen, The Netherlands;

${ }^{3}$ Expertise Center for Elderly Care,

Orbis Medical Center, Sittard,

The Netherlands; ${ }^{4}$ Institute Charles

Delaunay, Université de Technologie

de Troyes, Troyes, France
Correspondence: Joan Vermeulen School for Public Health and Primary Care (CAPHRI), Maastricht University, Duboisdomein 30, PO Box 616, 6200 MD, Maastricht, The Netherlands

$\mathrm{Tel}+3 \mid 433882199$

Fax +31433884162

Email j.vermeulen@maastrichtuniversity.nl
Purpose: To involve elderly people during the development of a mobile interface of a monitoring system that provides feedback to them regarding changes in physical functioning and to test the system in a pilot study.

Methods and participants: The iterative user-centered development process consisted of the following phases: (1) selection of user representatives; (2) analysis of users and their context; (3) identification of user requirements; (4) development of the interface; and (5) evaluation of the interface in the lab. Subsequently, the monitoring and feedback system was tested in a pilot study by five patients who were recruited via a geriatric outpatient clinic. Participants used a bathroom scale to monitor weight and balance, and a mobile phone to monitor physical activity on a daily basis for six weeks. Personalized feedback was provided via the interface of the mobile phone. Usability was evaluated on a scale from 1 to 7 using a modified version of the Post-Study System Usability Questionnaire (PSSUQ); higher scores indicated better usability. Interviews were conducted to gain insight into the experiences of the participants with the system.

Results: The developed interface uses colors, emoticons, and written and/or spoken text messages to provide daily feedback regarding (changes in) weight, balance, and physical activity. The participants rated the usability of the monitoring and feedback system with a mean score of 5.2 (standard deviation 0.90) on the modified PSSUQ. The interviews revealed that most participants liked using the system and appreciated that it signaled changes in their physical functioning. However, usability was negatively influenced by a few technical errors.

Conclusion: Involvement of elderly users during the development process resulted in an interface with good usability. However, the technical functioning of the monitoring system needs to be optimized before it can be used to support elderly people in their self-management.

Keywords: user-centered design, telemonitoring, physical functioning, community-dwelling elderly people, usability

\section{Introduction}

Disability, often defined as experienced difficulty in performing activities in any domain of life, poses a threat to independence of community-dwelling elderly people. ${ }^{1}$ Elderly people suffering from a decrease in certain indicators of physical functioning, such as gait speed, physical activity, weight, grip strength, balance, and lower extremity function, have an increased risk to develop disability. ${ }^{2-5}$ Elderly people and care professionals are often not aware of decreases in indicators of physical functioning at an early stage and decline can continue until (health) problems arise. ${ }^{6}$ Innovative technologies can play an important role in remote monitoring and early identification of elderly people who suffer from functional decline and therefore have an increased risk to develop disabilities. ${ }^{7,8}$ These people are the ones who are most likely to benefit 
from existing disability prevention programs. ${ }^{9,10}$ Furthermore, literature reviews reveal that such care technologies can also positively influence self-management, health behaviors, medical outcomes, and quality of life of elderly people. ${ }^{11,12}$

Researchers from Maastricht University (MU) in The Netherlands and engineers from the Institute Charles Delaunay at the Université de Technologie de Troyes (UTT) in France collaborated to develop an innovative telecare system that monitors indicators of physical functioning in elderly people over time and provides feedback regarding changes. The system consists of three devices: a bathroom scale for monitoring weight and balance, a Grip-ball for monitoring grip strength, and a mobile phone with a built-in accelerometer for monitoring physical activity. ${ }^{13-16}$ The devices are equipped with Bluetooth so that the information can be transferred automatically to the mobile phone. Via the interface of the mobile phone, feedback can be provided to the users regarding changes in their physical functioning; this can support them in their self-management. Furthermore, the mobile phone can transfer the data to a database that is accessible to care professionals who can use the information to provide pro-active care to their patients. The monitoring and feedback system is depicted in Figure 1.

Previous research has shown that it is important that telecare technologies, such as the monitoring and feedback system described above, should meet the needs and preferences of the users. ${ }^{17,18}$ Furthermore, usability remains a critical issue in such telecare technologies, ${ }^{11}$ because if elderly users are not able to use the monitoring system and mobile phone properly or do not understand the feedback that is presented via the mobile interface, it is unlikely that the system will be used to support self-management. Involving end-users during the development of care technologies can ensure that the technology meets the needs and preferences of the users and it can improve the usability of the technology. ${ }^{19-21}$ Therefore, the objective of this study was to involve elderly people during the development of the mobile interface of the monitoring and feedback system and to test the monitoring and feedback system in real life.

\section{Material and methods}

The methods section is divided into two parts. The first part focuses on the methods that were used during the different phases of the user-centered development process. The second part focuses on the methods that were used during the pilot study.

\section{Part I: methods user-centered development process}

The user-centered development process of the interface consisted of the following phases: (1) selection of user

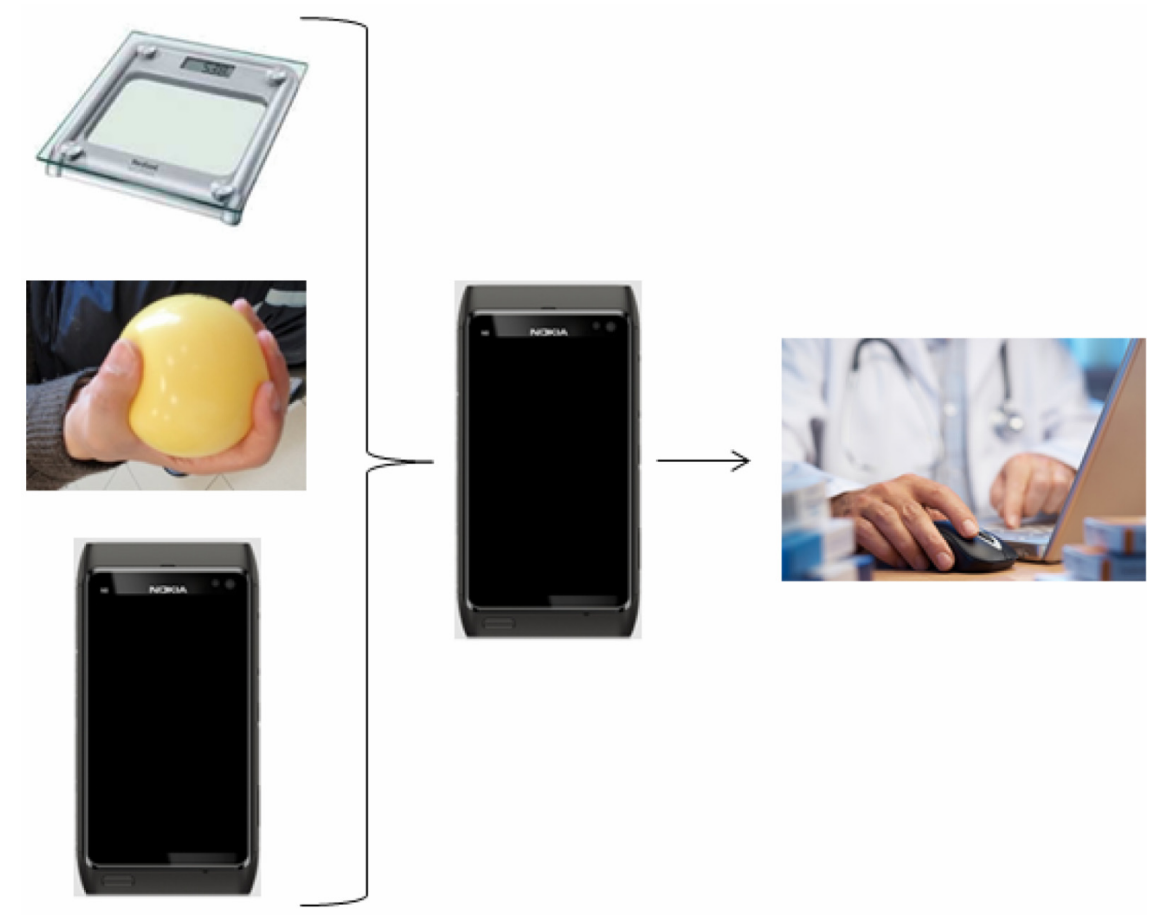

Figure I Monitoring and feedback system.

Notes: The monitoring and feedback system consists of three devices: a bathroom scale that measures weight and balance, a Grip-ball that measures grip strength, and a mobile phone with a built-in accelerometer that measures physical activity. Weight, balance and grip strength data are transferred to the mobile phone via Bluetooth. Via the screen of the mobile phone, feedback regarding (changes in) the physical indicators is provided to the elderly user. Besides that, all measurements are forwarded to a database that is accessible to care professionals. 
representatives; (2) analysis of users and their context; (3) identification of user requirements; (4) development of the interface; and (5) evaluation of the interface in the lab. Figure 2 provides an overview of the methods that were used during the different phases of the development process.

A key principle of user-centered design is that the process should be iterative. ${ }^{22}$ This was the case in the development process because the different phases, often referred to as iterations, were repeated until the desired result was obtained. Each phase delivered the input for the next phase and at the end of each phase the development team checked whether the results were compatible with those of the previous phase. Other key principles of user-centered design, such as active user involvement and working in multidisciplinary teams were followed as well throughout the development process to ensure that the interface would fit the preferences and

\section{Phase 1: Selection of user representatives}

- Three user representatives volunteered to be part of the development team during a meeting that was organized by the House for Care

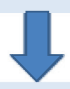

Phase 2: Analysis of users and their context

- A literature search was conducted regarding characteristics of elderly users of mobile interfaces

- Four discussion group meetings were organized with a geriatrician, geriatric nurse, geriatric physiotherapist, nursing home physician, and social gerontologist

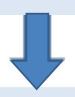

Phase 3: Identification of user requirements

- Three user-group meetings were organized with the user representatives and their advisor

- Community-dwelling elderly people provided their input during a workshop

Phase 4: Development of the interface

- First prototype of the interface was developed by technical engineers of UTT based on the user requirements formulated in phase 3

- Second prototype was developed based on the new and unmet requirements that were identified

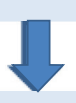

Phase 4: Evaluation of the usability of the interface in-lab

- Heuristic evaluation second prototype by non-users

- Usability evaluation third prototype by 11 elderly users via think aloud procedure and adapted version of the PostStudy System Usability Questionnaire (PSSUQ)

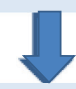

Development of final prototype of the interface

(Based on the results of the usability evaluation by users)

\section{Review first prototype}

Elderly representatives and their advisor reviewed the first prototype and identified new and unmet requirements that guided the development of the second prototype

\section{Adjust second prototype}

The third prototype is developed based on results of heuristic evaluation

Figure 2 User-centered development process. 
requirements of the potential users. This will be illustrated in the next paragraphs.

\section{Phase I: selection of user representatives}

Three elderly user representatives, all men aged over 65 years old, volunteered to be part of the development team after attending a meeting during which several researchers presented their projects. This meeting was organized by the House for Care (in Dutch: "Huis voor de Zorg") which is an independent organization that advocates patient empowerment and is committed to defending the interests and increasing the say and control of patients and other users of care. Together with researchers from MU and engineers from UTT, these representatives and their advisor from the House for Care formed the development team throughout the entire process.

\section{Phase 2: analysis of users and their environmental context}

A literature search was performed to gain more insight into the characteristics of community-dwelling elderly people that are relevant to the development of a mobile interface. Furthermore, a geriatrician, geriatric nurse, geriatric physiotherapist, nursing home physician and social gerontologist advised the development team during four discussion meetings regarding important characteristics of potential users of the interface that provides feedback about (changes in) physical functioning.

\section{Phase 3: identification of user requirements}

Three user-group meetings were organized with the elderly representatives from the House for Care and their advisor during which they provided their input regarding the user requirements to the researchers from MU. The first user-group meeting focused on the feedback and the second meeting focused on the mobile interface. During the third user-group meeting the information from the two previous user-group meetings was discussed and prioritized, which resulted in a list of requirements.

Furthermore, 24 elderly people who attended regular social gatherings organized by the Catholic Association for Elderly People participated in a workshop that took place during one of their gatherings. The monitoring system was presented to them and the requirements for the interface, as identified previously by the user representatives, were explained. The participants of the workshop were invited to discuss whether they liked and agreed with these requirements or not. New ideas or requirements were added to the list of requirements.

\section{Phase 4: development of the interface}

The list of requirements that resulted from Phase 3 was used as the foundation for the development of the first prototype of the interface. This prototype was developed by engineers from UTT. During a fourth user-group meeting, the user representatives and their advisor explored whether their requirements were met by the first prototype of the interface. Unmet or new requirements were added to the list of requirements and were used to guide the development of the second prototype of the interface.

\section{Phase 5: evaluation of the usability of the interface in the lab}

The usability of the interface was evaluated first in a heuristic evaluation by non-users. After that it was evaluated by potential elderly users via a think aloud procedure.

\section{Heuristic evaluation by non-users}

Three system developers and five non-experts evaluated the second prototype in a heuristic evaluation. The evaluators checked whether the following ten heuristics were violated while clicking through the interface: visibility of system status, match between system and real world, user control and freedom, consistency and standards, error prevention, recognition rather than recall, flexibility and efficiency of use, aesthetic and minimalist design, help users recognize/diagnose/recover from errors, and help and documentation. ${ }^{23,24}$ Identified violations were remedied in the third prototype of the interface.

Think aloud usability test by users

Elderly people who participated in the workshop in phase 3 were invited to evaluate the third prototype of the interface using a think aloud procedure. Participants individually performed eleven tasks using the mobile interface after receiving general instructions. They provided feedback on the process and indicated what caused difficulties by thinking aloud. Participants were observed by a trained observer who registered whether the participants made mistakes during the tasks and whether they needed assistance. The observers also ensured that all the "think aloud" comments of the participants were registered.

Furthermore, the participants evaluated the usability of the interface using a questionnaire that contained translated 
items from the Post-Study System Usability Questionnaire (PSSUQ) ${ }^{25} \mathrm{~A}$ few items of the original PSSUQ were replaced by items regarding specific features of the interface. Each item of the questionnaire was rated on a scale from 1 (I strongly disagree) to 7 (I strongly agree) and participants could explain their rating in the free text space. Examples of the items were: "Overall, I am satisfied with how easy it is to use this system"; "The information that was provided on the screen of the mobile phone was clear"; and "The menu on the mobile phone has all functions I expect it to have". The modified version of the PSSUQ that was used during the think aloud procedure is presented in Supplementary material.

Means and standard deviations were calculated to determine the usability of the interface; higher scores indicated better usability. Results from the think aloud procedure and PSSUQ were used to develop the fourth and final prototype.

\section{Part 2: methods pilot study Design and participants}

The final prototype of the mobile interface was integrated into the monitoring system so that the system could provide feedback to the user. Originally, the monitoring system consists of three devices: a bathroom scale, a Grip-ball, and a mobile phone; but, unfortunately the Grip-ball could not be included in the pilot study due to problems in its production process. Therefore, a system consisting of the bathroom scale and mobile phone was tested during the pilot study with 6-week follow-up.

Participants were recruited via the geriatric outpatient clinic at Orbis Medical Center in Sittard, The Netherlands. Inclusion criteria were: 70 years or older, communitydwelling, mobility or functional problems, cognitively not impaired (Mini Mental State Examination [MMSE] >23), able to step onto a bathroom scale independently, and willing to learn how to use the interface of the mobile phone. Exclusion criteria were: planned admission to a nursing home/hospital during the study, being confined to bed, serious visual or hearing impairments, and contra indication for physical exercise. Eight patients were invited by their geriatrician and received an information letter and a consent form via mail. The researcher contacted them after two weeks to ask whether they were willing to participate and whether they had any questions. Patients who decided to participate provided written informed consent. This pilot study was approved by the Medical Ethical Committee Atrium Orbis Zuyd (NL35961.096.11).

\section{Procedure}

Participants were instructed to step onto the bathroom scale every day between 7:00 and 10:30 to monitor their weight and balance. Feedback regarding (changes in) these indicators was provided to the user via the mobile interface directly after the measurement. To monitor their physical activity, the users were instructed to wear the phone with them in their pocket or on a belt. Participants monitored their activity from the morning until after 20:00 and received feedback about (changes in) their activity directly after pressing "stopactivity monitoring". After that, they were instructed to put the phone back in the docking-station for charging.

More detailed information about the content and presentation of the feedback and feedback algorithm is provided in the last paragraph of part 1 of the results section where the final prototype that was developed will be explained.

\section{Measurements}

After 3 weeks, the participants received a modified version of the PSSUQ. ${ }^{25}$ This modified version differed from the one that was used during the think aloud procedure. Some items were removed from the version of the PSSUQ that is presented in Supplementary material because they were not applicable and some questions that focused on the usability of the bathroom scale were added. As a result, the items of the modified PSSUQ that was used during the pilot study could be divided into three subscales: usability of the bathroom scale (5 items), usability of the mobile phone (10 items), and usability of the system as a whole (10 items). The participants rated each item on a scale from 1 to 7 ; higher scores indicated better usability. Besides that, free space was available after each question so that the participant could provide an explanation or clarification. Examples of the items were: "I liked using the bathroom scale daily to measure my weight and balance"; "I needed a lot of help with using the mobile phone"; "I liked using the monitoring system"; and "Overall I am satisfied with the monitoring system".

After 6-week follow-up, semi-structured interviews were conducted to gain insight into the experiences of the participants with the system. Topics discussed were: use of the bathroom scale and the mobile phone, feedback messages, satisfaction with the system, and usefulness of the system on a larger scale in the future.

Finally, all weight, balance, and activity measurements were registered automatically using the mobile phone. These data were used to study the adherence to the daily monitoring regimen. 


\section{Analyses}

Firstly, mean usability scores (standard deviation [SD]) were calculated for the total modified PSSUQ and its three subscales. Besides that, usability sumscores were calculated for each participant separately, higher scores indicated better usability. Secondly, the interviews were transcribed verbatim and analyzed using Nvivo version 9.0 (QSR International Limited; Warrington, Cheshire, UK). Finally, adherence to the daily monitoring regimen was calculated by counting the number of days that data on all three physical indicators (weight, balance, and activity) were saved by the mobile phone and dividing this by the total number of days that a participant was included in the pilot study.

\section{Results}

The results section is divided into two parts. The first part focuses on the results that were obtained during the phases of the user-centered development process. The second part focuses on the results of the pilot study.

\section{Part I: results user-centered development process}

\section{Users and their environmental context}

The literature study revealed that elderly people more often suffer from loss of cognitive capacities, sight loss, hearing loss, and decreased motor skills than younger people. These restrictions can cause difficulty with the use of small screen mobile interfaces. ${ }^{26-28}$ Therefore, it is important to take possible cognitive, sensory, and motor restrictions into account in developing an interface that provides feedback to elderly people regarding their own physical functioning..$^{29}$ Attention should be paid to factors such as screen size, font size, density of information, and level of contrast. ${ }^{30-33}$ This can prevent usability problems. ${ }^{34,35}$

Advice from the professionals during the discussion meetings revealed that elderly people who are at risk for, or already suffer from, decreased physical functioning should be the users of the interface. They are the ones who can benefit from the feedback messages supporting self-management. An important remark was that we should be very careful with the feedback that is provided because feedback about decreases in physical functioning can be very confronting to elderly users. Elderly people should not become "scared" of the feedback as this will cause (unnecessary) distress and might result in discontinued use of the system. Furthermore, the professionals stressed the importance of the social environment and the facilitating role that spouses, children, or neighbors can play in learning how the interface works and in understanding the feedback messages. This was later confirmed by elderly people during the user-group meetings and workshop.

\section{Identification of user requirements}

The requirements that were identified during the user-group meetings and workshop are presented in Table 1. The requirements that were identified by the user representatives and their advisor after reviewing the first prototype are included in the table as well.

\section{Usability of the interface in the lab}

Violations of heuristics identified by non-users

The heuristic evaluation revealed some consistency violations in the second prototype of the interface. Arrows were used on the interface to indicate whether a certain physical indicator had increased, decreased or stayed the same, but the use of the arrows was not consistent across the different indicators. Therefore, the arrows were deleted from the interface in the third prototype. There was also a mismatch between the color, emoticon, and the text on some of the screens. Another heuristic that was violated according to some evaluators was the user language heuristic. In some cases, words that were used on the buttons were not common and therefore difficult to understand for the potential end-users. An example is the word "parameter". These violations were remedied in the third prototype of the interface.

\section{Usability of interface in think aloud procedure}

Four men and seven women with a mean age of 79 years (SD 5.0 years) participated in the think aloud evaluation

\section{Table I User requirements}

User requirements identified during user-group meetings
and workshop
Feedback regarding 3 or 4 indicators is enough
Information should be easy to obtain
Use of one button per indicator
Menu with just a few layers
Feedback that is easy to understand
Feedback should be fun to watch (eg, by using colors and pictures)
Easy overview of the values and changes of the indicators
There should be interaction between the user and the interface
Possibility of receiving spoken feedback instead of written feedback
Touch screen to avoid problems with navigating through the interface
and pressing buttons
Large buttons on a touch screen
Use of large letter type
Docking station for charging the phone
User requirements added after reviewing the first prototype
Layout should be changed so that the screen is used optimally
Letters should be bigger
Information on the screen should be organized differently (instead
of four quadrants five buttons in a list should be used)


of the third prototype of the interface. Most participants had never used a phone with a touch screen before. Some of them had experience with regular mobile phones. Their comments during the tasks indicated that some buttons on the interface were too small and other buttons did not attract sufficient attention and were overlooked. Furthermore, some participants preferred bigger letters and others preferred black text instead of white text.

In addition to the think aloud comments, the observers noticed that most participants handled the phone correctly but that they had to get used to the touch screen. Sometimes, they needed to press the same button a few times before it would respond, but most participants became accustomed to the touch screen after practicing a few times. Most participants sought confirmation from the observer at the beginning of the session, but their confidence seemed to increase after successful completion of the first few tasks.

The mean usability score on the modified version of the PSSUQ was 5.90 (SD 1.1). One participant gave a mean score of 3.7 whereas the other 10 participants all gave mean usability scores of 5.6 or higher.

\section{The final version of the developed interface}

Figure 3 shows three screenshots of the developed interface. The application consists of three layers. The screenshot on the left shows the first layer which is the start screen of the interface consisting of five buttons; one for each physical indicator that users receive feedback on and one for changing the settings (eg, volume, text color etc). When users touch one of the first four buttons in the left screenshot, for example the "balance" button, they enter the second layer of the application which is represented by the middle screenshot. In the middle screenshot, users receive feedback regarding the measurement they performed today and how this relates to their personal goal which is based on their previous measurements. When the "history" button on the middle screenshot is touched, users enter the third layer of the application and the screenshot on the right appears. In the right screenshot an overview is provided of the last six balance measurements. Users can change the overview period to 2 weeks, 1 month, 3 months, or 6 months by touching the bar above the graph.

An automated feedback algorithm is responsible for the content of the feedback that is provided. To personalize the feedback, a "baseline profile" for each indicator is defined for each participant based on the mean and variation of the measurements during the first two weeks that they use the system. During these first two weeks, participants receive neutral feedback: the value of their measurement is presented on the mobile interface with a grey background. The feedback that participants receive after the first two weeks is personalized by comparing the daily measurements with the "baseline profile" also referred to as "goal" in the application. This comparison reveals whether each of the physical indicators increased, decreased, or remained the same. When changes are positive or when an indicator is stable, positive feedback messages are provided combined with a green background and happy smiley like in the middle screenshot of Figure 3. When indicators decrease, the feedback message explains how much the measurement differs from a participant's
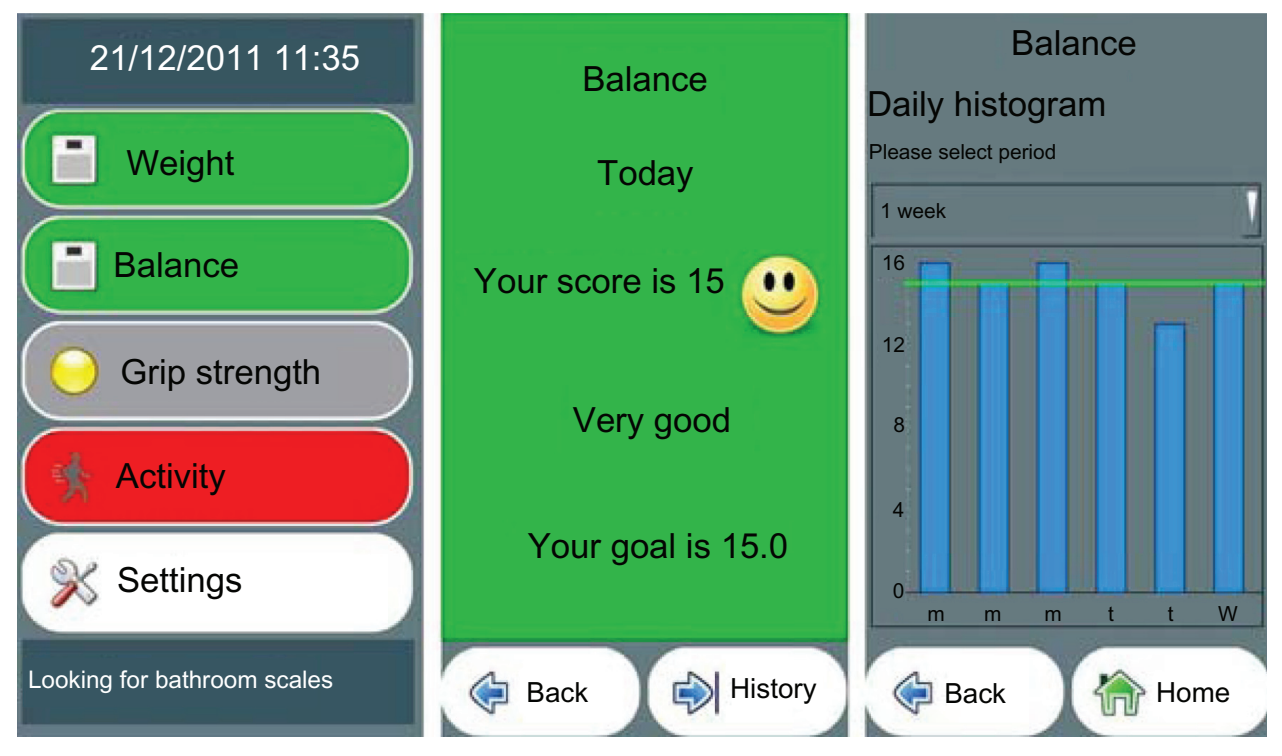

Figure 3 Three screenshots of the final interface. 
goal and this is combined with an orange or red background (depending on how much the indicator has decreased).

\section{Part 2: results pilot-study}

\section{Characteristics of included participants}

Two men (participants 2 and 3) and four women aged between 79 and 83 years old provided written informed consent. Participants 5 and 6 lived alone independently; the others lived together with their spouse. All participants visited the outpatient clinic of the geriatrician because they had fallen recently. All participants indicated at baseline that they owned a mobile phone that they only used sporadically. None of the participants had used a smartphone before. Participants 1, 2, 3, and 5 completed the pilot study and participated 42, 42, 42, and 37 days, respectively. Participant 4 decided to stop participation after using the monitoring system for 6 days; the main reason for her drop-out was that she did not feel supported enough by her husband. Participant 6 dropped out after two days because she was admitted to the hospital unexpectedly.

\section{Usability of the monitoring and feedback system}

The usability scores on the modified version of the PSSUQ are presented in Figure 4 for each participant separately. The mean overall usability score was 5.2 (SD 0.9) and scores varied between participants from 3.8 to 6.2. The mean scores of the subscales for the bathroom scale, mobile phone and system as a whole were 6.2 (SD 0.6), 5.0 (SD 0.8), and 4.8 (SD 1.0), respectively on a scale from 1 to 7 . The participant who dropped-out of the study after 6 days (participant 4) gave the lowest usability scores on all subscales.

\section{Experiences with the monitoring and feedback system}

The data that was gathered during the interviews was clustered into four themes: receiving feedback, use of the monitoring system, technical functioning of the devices, and use of the system in the future.

\section{Receiving feedback}

Most participants appreciated the feedback messages and indicated that these made them more aware of their own physical functioning. Example of a user response:

I appreciate that it signals changes. At a certain point you see that your weight drops from 81.5 to 78.6 . Then I think:

I have not been eating less than before. So then I will keep

an eye on that. (participant two, male, 80 years old)

When the feedback colors first appeared after two weeks of baseline monitoring with neutral feedback, some of the participants were confused because they forgot what was explained about these colors at the start of the study. They did not understand why the colors appeared and some participants even thought that this was an error of the mobile phone, so additional explanation from the researcher was needed. Despite this, the use of colors to provide feedback was appealing according to the participants. Example of a user response:

The device is alive. It is not a dead thing. It can change colors after a while. (participant five, female, 83 years old)

Furthermore all participants noticed that they sometimes received red feedback messages for balance and activity that should have been green. For example when a person with an activity goal of 35 minutes was active for 40 minutes and still received a red feedback message stating that activity had decreased. The participants experienced these incorrect red feedback messages as bothersome but it did not scare nor panic them. These wrong feedback messages resulted from a flaw in the feedback algorithm.

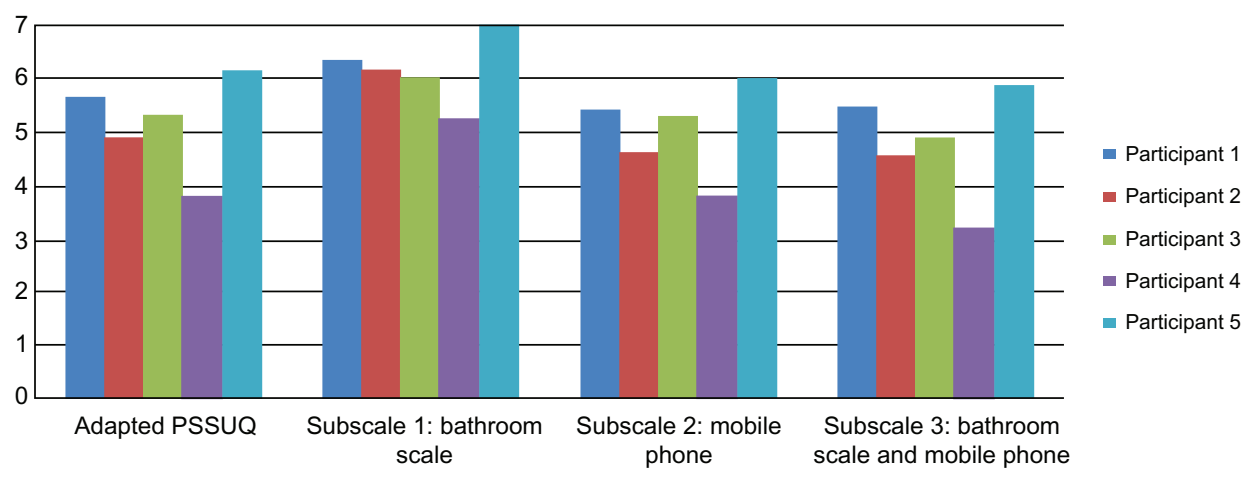

Figure 4 Mean usability scores per participant.

Abbreviation: PSSUQ, Post Study System Usability Questionnaire. 


\section{Use of the monitoring system}

Three participants indicated that they liked using the monitoring system every day and one was neutral. Using the monitoring system was not very difficult for them but one of the participants kept using the instruction manual every day while performing the measurements. Example of user response:

It is actually pretty easy... I press the buttons according to the manual, and then it's OK. (participant three, male, 79 years old)

Some aspects were mentioned that could improve the usability of the monitoring system. Firstly, the participants indicated that the "Start" and "Stop" button for the activity monitoring functionality should be separate buttons whereas during the study the "Start" button became the "Stop" button once activity monitoring was started. Secondly, all participants would appreciate a reset button that they can use when pressing a wrong button by accident on the touch screen. Thirdly, the female participants did not like the elastic waist band that they could use to carry the phone with them for activity monitoring on days that they wore clothes without pockets. They indicated that a less flexible belt would be more suitable to carry the weight of the mobile phone.

\section{Technical functioning}

Two types of technical errors occurred during the pilot study: on three occasions participants reported that the bathroom scale did not transfer the data to the mobile phone and on four occasions the participants reported that the application on the mobile phone had shut down automatically. Despite the fact that these errors did not occur often, they had a negative impact on the experiences of the participants with the monitoring system. Participants dealt with the errors differently. Some participants found a solution by looking in the instruction manual, others tried pushing different buttons but this did not solve the error, which frustrated them. Most participants called the researcher to report the error. In some cases the researcher visited the participant at home to solve the problem. Examples of user responses:

When everything works as it should, it's OK, then I am happy. Otherwise I panic a bit. (participant one, female, 84 years old)

When the screen with the buttons (the application) disappears, it is quite a fuss to get things back like they were. (participant three, male, 79 years old)

\section{Use of system in the future}

Participants think that self-monitoring physical functioning with the system could be useful to other elderly people. They especially like the idea that the mobile phone can send their measurements to a database where their care professionals can see them. Example of user response:

But I think it is a good system indeed that the doctor can use to keep an eye on things. (participant two, male, 80 years old).

The participants also identified possible difficulties/ obstacles for the use of the monitoring and feedback system in the future. For example: forgetfulness in elderly people can result in not using the system every day, or it might be difficult to learn how to use the monitoring system (due to lack of experience with computers/mobile phones or because of character traits), or elderly people might grow tired of using the system for a longer time.

\section{Adherence to daily monitoring regimen}

Frequency calculations revealed that participant 1, 2, and 5 did not use the bathroom scales and mobile phone on 7 days (17\%), 2 days (5\%), and 13 days (35\%) of the pilot study, respectively. Participant 3 used the monitoring and feedback system every day. No adherence rate was calculated for participants 4 and 6 because they dropped out of the study. The frequency data from the four participants who completed the pilot study resulted in an average adherence rate of $87 \%$ to the daily monitoring regimen.

\section{Discussion}

Mixed-methods were used and key principles of user-centered design were respected throughout the development process to ensure that the developed interface would meet the needs and preferences of the end-users. ${ }^{18,22}$ The involvement of elderly people during the development process resulted in a usable mobile interface that provides feedback regarding (changes in) indicators of physical functioning that is easy to understand. The interface that emerged from the user-centered development process was integrated in the monitoring and feedback system and tested in a pilot study. Participants of the pilot study were able to use the system and liked the feedback that was provided to them. The monitoring and feedback system satisfied most needs and preferences of the end-users and was considered easy-to-use which resulted in good adherence to the daily monitoring regimen. Previous research also shows that ease of use is a very important predictor of adherence to telecare systems in elderly persons with functional or mobility 
problems ${ }^{36}$ Only one participant had lower adherence during the pilot study, which was mainly caused by the fact that the participant could not restart the application by herself after it had shut down automatically. So, her low adherence was the result of an error in the application that caused a usability problem. The few technical errors that occurred during the pilot study annoyed the users and sometimes caused confusion. These errors need to be remedied before the system can be evaluated in a larger group since an important prerequisite for the uptake in technology in practice is that the monitoring system should operate without interruptions. ${ }^{11}$

A recent review by van den Berg et al regarding telemedicine and telecare for older patients revealed that the majority of studies in this field are carried out in "younger older patients" who do not always represent the target group of the innovation. ${ }^{37}$ A strength of this pilot study is that only "older patients" were included. Another advantage is that the monitoring and feedback system was tested in the daily lives of elderly people instead of in a controlled lab-situation; this provides more accurate and detailed information into the experiences and problems that can occur. ${ }^{38}$

The experiences of the participants with the monitoring and feedback system cannot be generalized due to the small study sample which is a limitation of the pilot study. Despite this, most usability problems were probably identified during the usability test in the lab and the pilot study since, according to Nielssen et al, five participants is sufficient to identify $80 \%$ of these problems. ${ }^{39}$ Another limitation of this study is that, although both based on the PSSUQ, different questionnaires were used to test the usability of the interface in lab during the development phase and to test the usability of the monitoring and feedback system during the pilot study and that both versions were not validated.

\section{Implications for clinical practice and future research}

In order to have an added value for community-dwelling elderly people and care professionals the monitoring and feedback system should not function as a stand-alone intervention but instead it should be integrated in usual care. Previous research has shown that blended-care approaches, where telecare interventions are embedded in professional care processes, yield more positive results and are more sustainable. ${ }^{40,41}$ However, before the monitoring and feedback system can be integrated in care, more insight is needed into its long-term acceptance according to elderly users and their care providers. Therefore, the monitoring system and interface are currently being improved based on the results of the pilot study. Subsequently, a 6-month follow-up study is being organized during which community-dwelling elderly people will use the improved system including the Grip-ball. This follow-up study will not only focus on the experiences of the elderly users, but also on the care professionals who will use the database to monitor their patients from a distance. We expect that the follow-up study will also provide more insight into the possibility of detecting clinically relevant changes in physical functioning with the devices of the monitoring system.

\section{Acknowledgments}

The authors would like to thank the representatives and advisors from the House for Care for their collaboration throughout this research project. Furthermore, the authors would like to thank all the members of the Catholic Association for Elderly People who participated in the workshop and/or the final in-lab evaluation of the usability of the interface. Also, the authors would like to thank the professionals for their input during the development process. Emmanuel Menard, Luc Rodrigues, Michael Mordefroy, and Pauline Hourseau from the Université de Technologie de Troyes are acknowledged for their contributions to the development of the interface and their technical support during the pilot study. Finally, the authors would like to thank the people who participated in the pilot study and the nurse practitioner of the Expertise Center for Elderly Care of the Orbis Medical Center in Sittard for her help in recruiting the participants.

\section{Disclosure}

The authors report no conflicts of interest in this work.

\section{References}

1. Jette AM. Toward a common language for function, disability, and health. Phys Ther. 2006;86(5):726-734.

2. Vermeulen J, Neyens JC, van Rossum E, Spreeuwenberg MD, de Witte LP. Predicting ADL disability in community-dwelling elderly people using physical frailty indicators: a systematic review. $B M C$ Geriatr. 2011;11:33.

3. Rothman MD, Leo-Summers L, Gill TM. Prognostic significance of potential frailty criteria. J Am Geriatr Soc. 2008;56(12):2211-2116.

4. Gill TM, Allore H, Holford TR, Guo Z. The development of insidious disability in activities of daily living among community-living older persons. Am J Med. 2004;117(7):484-491.

5. Balzi D, Lauretani F, Barchielli A, et al. Risk factors for disability in older persons over 3-year follow-up. Age Ageing. 2010;39(1):92-98.

6. Besdine RW, Wetle TF. Improving health for elderly people: an international health promotion and disease prevention agenda. Aging Clin Exp Res. 2010;22(3):219-230.

7. Boulos MN, Wheeler S, Tavares C, Jones R. How smartphones are changing the face of mobile and participatory healthcare: an overview, with example from eCAALYX. Biomed Eng Online. 2011;10:24.

8. Patrick K, Griswold WG, Raab F, Intille SS. Health and the mobile phone. Am J Prev Med. 2008;35(2):177-181. 
9. Gitlin LN, Winter L, Dennis MP, Corcoran M, Schinfeld S, Hauck WW A randomized trial of a multicomponent home intervention to reduce functional difficulties in older adults. J Am Geriatr Soc. 2006;54(5): 809-816.

10. Worm CH, Vad E, Puggaard L, Stovring H, Lauritsen J, Kragstrup J. Effects of a multicomponent exercise program on functional ability in community-dwelling frail older adults. J Aging Phys Act. 2001;9(4): 414-424.

11. Botsis T, Hartvigsen G. Current status and future perspectives in telecare for elderly people suffering from chronic diseases. J Telemed Telecare. 2008;14(4):195-203.

12. Koch S, Hägglund M. Health informatics and the delivery of care to older people. Maturitas. 2009;63(3):195-199.

13. Vermeulen J, Neyens JCL, Spreeuwenberg MD, et al. Construct validity of a modified bathroom scale that can measure balance in elderly people. J Am Med Dir Assoc. 2012;13(7):665. e1-e5.

14. Duchêne J, Hewson DJ. Longitudinal evaluation of balance quality using a modified bathroom scale: usability and acceptability. JTelemed Telecare. 2011;17(8):421-426.

15. Hewson DJ, Li K, Frerejean A, Hogrel JY, Duchene J. Domo-Grip: functional evaluation and rehabilitation using grip force. Conf Proc IEEE Eng Med Biol Soc. 2010;2010:1308-1311.

16. Hewson DJ, Duchêne J, Charpillet F. The PARAChute project: remote monitoring of posture and gait for fall prevention. EURASIP Journal on Advances in Signal Processing. Volume 2007, issue 1, start \& end page 027421 .

17. Teixeira L, Ferreira C, Santos BS. User-centered requirements engineering in health information systems: a study in the hemophilia field Comput Methods Programs Biomed. 2012;106(3):160-174.

18. Kujala S. User involvement: a review of the benefits and challenges. Behav Inf Technol. 2003;22(1):1-16.

19. van Gemert-Pijnen JE, Nijland N, van Limburg M, et al. A holistic framework to improve the uptake and impact of eHealth technologies. J Med Internet Res. 2011;13(4):e111.

20. De Vito Dabbs A, Myers BA, Mc Curry KR, et al. User-centered design and interactive health technologies for patients. Comput Inform Nurs. 2009;27(3):175-183.

21. De Rouck S, Jacobs A, Leys M. A methodology for shifting the focus of e-health support design onto user needs: a case in the homecare field. Int J Med Inform. 2008;77(9):589-601.

22. Gulliksen J, Göransson B, Boivie I, Blomkvist S, Persson J, Cajander Å. Key principles for user-centered systems design. Behav Inf Technol. 2003;22(6):379-409.

23. Nielsen J. Usability Engineering, 1st ed. Cambridge: AP Professional; 1993.

24. Nielsen J, Mack RL, editors. Usability Inspection Methods, 1st ed. New York: John Wiley and Sons; 1994.

25. Lewis JR. Psychometric evaluation of the PSSUQ using data from five years of usability studies. Int J Hum Comput Interact. 2002;14(7): 463-488.
26. Gregor P, Newell AF, Zajicek M. Designing for Dynamic Diversity: Interfaces for Older People. Assets '02 Proceedings of the fifth international ACM conference on assistive technologies. New York, NY: ACM; 2002:151-156.

27. Edlin-White R, Cobb S, D’Cruz M, Floyde A, Lewthwaite S, Riedel J. Accessibility for older users through adaptive interfaces: opportunities, challenges and achievements. In: Jacko JA, editor. Human-Computer Interaction. Towards Mobile and Intelligent Interaction Environments. City: Springer Berlin Heidelberg; 2011:483-489.

28. Lorenz A, Oppermann R. Mobile health monitoring for the elderly: designing for diversity. Pervasive Mob Comput. 2009;5(5):478-495.

29. Hawthorn D. Possible implications of ageing for interface designers. Interact Comput. 2000;12(5):507-528.

30. Darroch I, Goodman J, Brewster S, Gray P. The Effect of Age and Font Size on Reading Text on Handheld Computers. INTERACT '05 Proceedings of the 2005 IFIP TC13 international conference on HumanComputer Interaction. Berlin: Springer-Verlag; 2005:253-266.

31. Zhou J, Rau PLP, Salvendy G. Use and design of handheld computers for older adults: a review and appraisal. Intern J Hum-Comput Int. 2012;28(12):799-826.

32. Sanchez CA, Goolsbee JZ. Character size and reading to remember from small displays. Comp and Educ. 2010;55(3):1056-1062.

33. Sweeney S, Crestani F. Effective search results summary size and device screen size: is there a relationship? Inform Process Manag. 2006;42(4) 1056-1074.

34. Holzinger A, Searle G, Nischelwitzer A. On some aspects of improving mobile applications for the elderly. In: Stephanidis C, editor Universal access in human computer interaction: coping with diversity. Heidelberg: Springer; 2007:923-932.

35. Ziefle M. Information presentation in small screen devices: the trade-off between visual density and menu foresight. Appl Ergon. 2010;41(6): 719-730.

36. Wade R, Cartwright C, Shaw K. Factors relating to home telehealth acceptance and usage compliance. Risk Manag Healthc Policy. 2012;5:25-33.

37. van den Berg N, Schumann M, Kraft K, Hoffmann W. Telemedicine and telecare for older patients - a systematic review. Maturitas. 2012;73(2):94-114.

38. Kangas E, Kinnunen T. Applying user-centered design to mobile application development. Communications of the ACM. Designing for the mobile device. 2005:48(7); 55-59.

39. Nielsen J, Landauer TK. A mathematical model of the finding of usability problems. In: Arnold B, Van der Veer G, White T, editors. Human factors in computing systems. New York: ACM; 1993:206-213

40. May CR, Finch TL, Cornford J, et al. Integrating telecare for chronic disease management in the community: what needs to be done? BMC Health Serv Res. 2011;11:131.

41. Weinstein RS, Lopez AM, Krupinski EA, et al. Integrating telemedicine and telehealth: putting it all together. Stud Health Technol Inform. 2008;131:23-38. 


\section{Supplementary material}

Modified version Post Study System Usability Questionnaire (PSSUQ) used during think aloud procedure.

Each item on the questionnaire was rated on a 7-point scale from 1 (I strongly disagree) to 7 (I strongly agree).

$\begin{array}{llllllllr}\begin{array}{l}\text { I strongly } \\ \text { disagree }\end{array} & 1 & 2 & 3 & 4 & 5 & 6 & 7 & \text { I strongly } \\ \text { agree }\end{array}$

1. Overall, I am satisfied with how easy it is to use this system.

2. It was easy to switch on the mobile phone.

3. It was easy to connect the adapter to the mobile phone.

4. The menu on the mobile phone was easy to use.

5. I could complete the tasks effectively using the mobile phone.

6. I was able to complete the tasks quickly using the mobile phone.

7. I was able to complete the tasks efficiently using the mobile phone.

8. I felt comfortable using the mobile phone.

9. It was easy to learn to use the mobile phone.

10. Whenever I made a mistake using the mobile phone, I could recover easily and quickly.

11. The information that was provided on the screen of the mobile phone was clear.

12. It was easy to find the right information on the mobile phone.

13. The information provided on the screen of the mobile phone was easy to understand.

14. The information on the screen of the mobile phone was effective in helping me complete the tasks.

15. The organization of the information on the screen of the mobile phone was clear.

16. The interface of the mobile phone looked pleasant.

17. I liked using the interface of the mobile phone.

18. The menu on the mobile phone has all functions I expect it to have.

19. Overall, I am satisfied with the mobile phone.

20. The letters and words on the screen of the mobile phone were easy to read.

21. The contrast of the colors on the screen of the mobile phone was good.

22. The images on the screen were clearly visible.

23. Next week, I will remember as well as now how the mobile phone works.

Patient Preference and Adherence

Dovepress

\section{Publish your work in this journal}

Patient Preference and Adherence is an international, peer-reviewed, open access journal focusing on the growing importance of patient preference and adherence throughout the therapeutic continuum. Patient satisfaction, acceptability, quality of life, compliance, persistence and their role in developing new therapeutic modalities and compounds to optimize clinical outcomes for existing disease states are major areas of interest. This journal has been accepted for indexing on PubMed Central. The manuscript management system is completely online and includes a very quick and fair peer-review system. Visit http://www.dovepress.com/ testimonials.php to read real quotes from published authors. 